\title{
Analyzing Crime Networks: A Complex Network-Based Approach
}

\section{Husam B. Sultan}

husam.csp78@student.uomosul.edu.iq

Department of Computer Science

College of Computer Science and Mathematics

University of Mosul, Mosul, Iraq

Received on: $27 / 08 / 2020$

Accepted on: 14/09/2020

\begin{abstract}
This article analyzed the crime network of Nineveh province based on the concepts of Complex Networks. To this end, two networks were created; the first represented the crimes that were committed in Nineveh province, while the second was the network of crime regions. These two networks were visualized and then analyzed using network centrality measurements. The results showed that several pairs of crimes had strong relations to each other. Moreover, it was found that some crime regions were considered as the core of crimes in the province. The results also showed that few regions were considered as the most dangerous parts of the province and they had strong tendencies to replicate their behaviour to other regions. Finally, the authors believe this is the first kind of works that take the crime network of Nineveh province as a case study.
\end{abstract}

Keywords: Crime Networks, Complex Networks, Nineveh Province.






\section{1.}

تعدُّ الجريمة من الأفعال السلبية التي تؤثر في الفرد خصوصاً والمجتمع عموماً [1]، وتعدُ أيضاً من العوامل

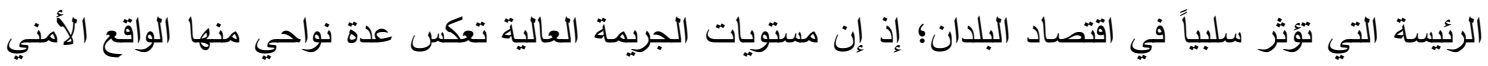

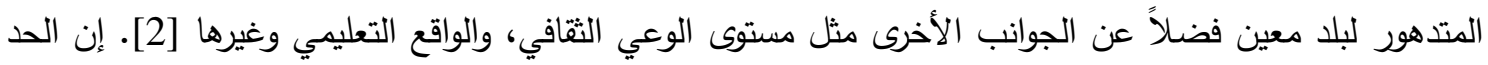
من مستوى الجرائم يقع على عاتق الجهات الأمنية أساساً وذلك بفرض القانون، ويقع أيضاً على عاتق المواطن من والى ناحية الالتزام بالقوانين واتباعها.

إن الحد من مستوى الجريمة يُمكن أن يكون بالتحليل الدقيق لثبكات الجريمة والوقوف على أبرز خواصها، ويُمكن بعد ذلك اعتماد نتائج التحليل في تحديد مواطن الخلل التي تحد من مستوى الجريمة [3]. يمكن إجراء التحليل على الثبكات الإجرامية بالطرائق الإحصائية التقليدية [4]، لكن ظهرت مؤخراً طرائق في مجال علوم

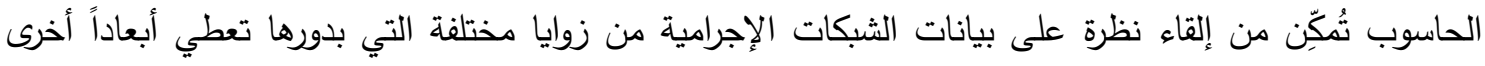

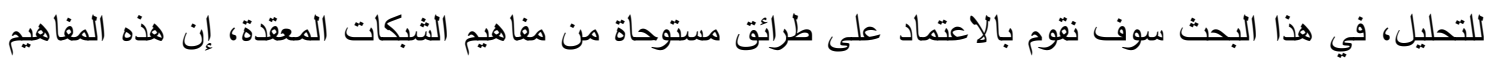
تعتمد أساساً على ثلاثة جوانب تخصصية، وهي علم الاجتماع، وعلم الحاسوب، وعلم الإحصاء، لتكوين شبكة

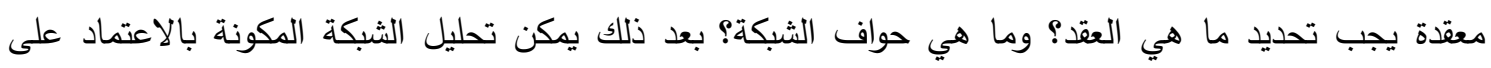
معايير معينة سوف تذكر لاحقاً في هذا البحث. استتاداً إلى اطلاعنا المستفيض على الدراسات المقدمة في مجال الجريمة في العراق وخارجه، والدراسات السابقة (كما سوف نرى لاحقاً)، تبين أن هناك نقصاً حاداً في أدب الجريمة لبحوث تلتناول حالة البلدان النامية

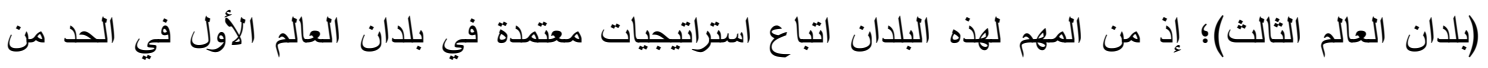
الجريمة، وذلك لتحسين مستوى تلك البلدان من الناحية الاقتصادية أولاً، وكذلك لتوفير بيئة آمنة لسكان تلك الكئك الدول. في هذا البحث سوف نقوم بتحليل شبكة الجريمة في محافظة نينوى شمالي العراق على وفق مفاهيم وأساسيات

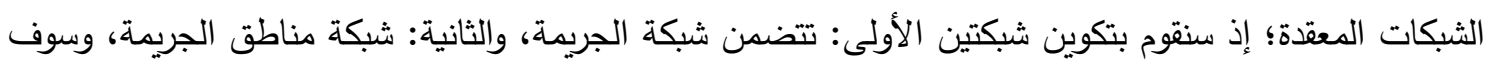

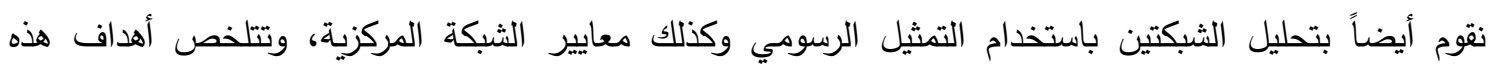
الدراسة بما يأتي: أ. كثف العلاقات ما بين الجرائم والتقصي عن وجود علاقات بينها لما له من أهمية في الحد من مستوى

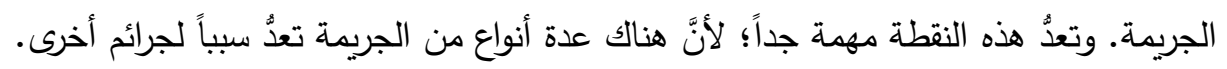
ب. تحليل مناطق الجريمة على أساس الجرائم التي تقع فيها وعلاقاتها مع مناطق الجريمة الأخرى في المحافظة. ت. التقصي عن المناطق الأخطر في ارتكاب الجريمة وتصديرها في المحافظة ومدينة الموصل تحديداً.



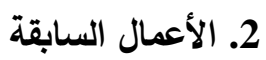

إن تحليل الثبكات الإجرامية باستخدام مفاهيم الثبكات المعقدة أصبح معتمداً في الكثير من الدول المتقدمة

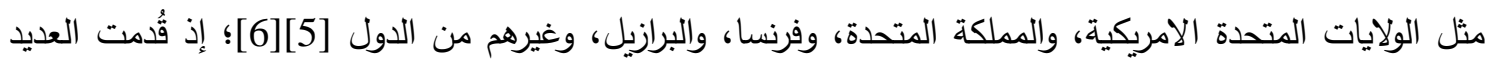

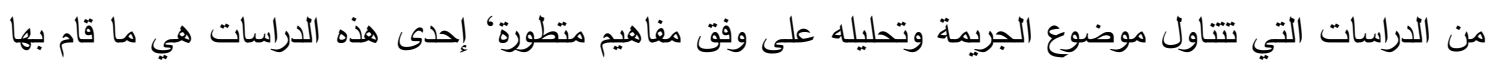
وايت وآخرون [7]؛ إذ قاموا بدراسة الخصائص المكانية للجريمة؛ وذلك عن طريق تكوين شبكة معقدة مكانئ مانية تعتئمد في ربط العقد على المسافات بين مواقع الجريمة، وقد استتنج الباحثون أن هناك علاقة بين مكان جريمة وأخرى، 
وقد اعتمد الباحثون على معلومات الجرائم المرتكبة في الولايات المتحدة الامريكية للسنوات 2007 إلى 2010،



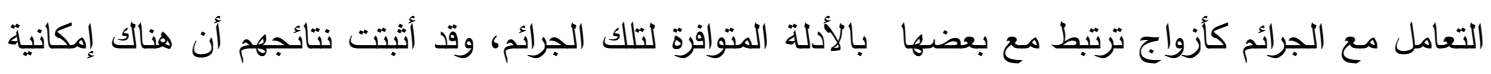

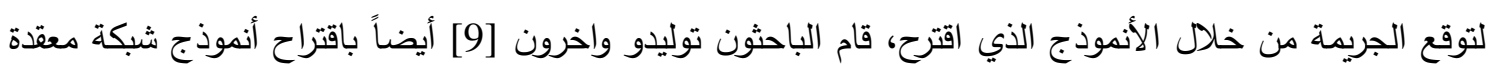



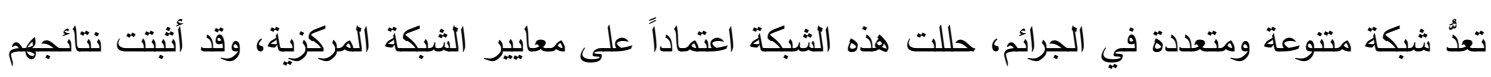

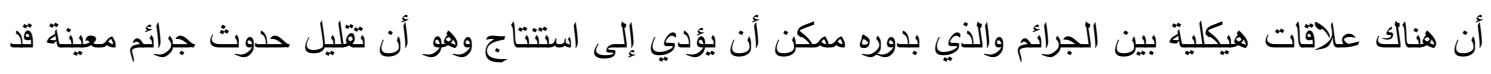



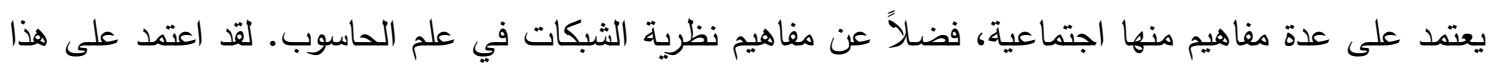
الأموذج في تعقب المجرمين من خلال الخصائص التي توفرها الثبكة التي تعتمد على مزيج من المفاهيم التي

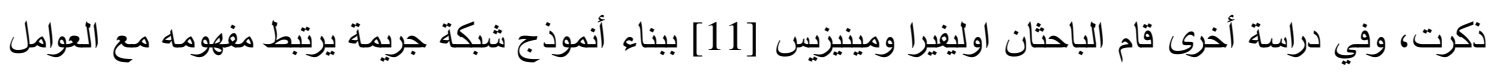

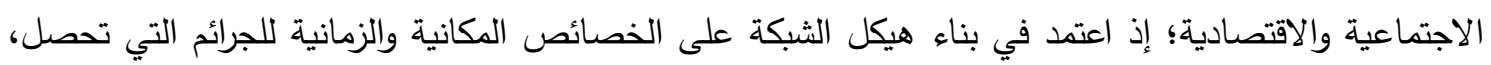
وقد توصل إلى أن أماكن حصول الجرائم لا يرتبط كثيراً مع حجم المدن التي تحدث فيهاء تلك الجرائم، لكئم لكن كثرة

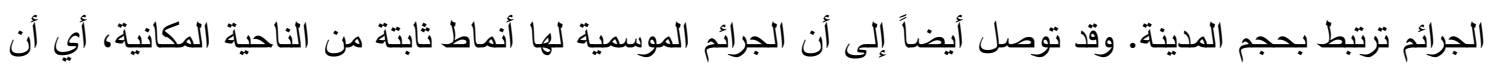
الجرائم التي تحدث في أماكن معينة في مواسم معينة تكون احتمالية تكرارها كبيرة جداً من ناحية الزمان والمكان،



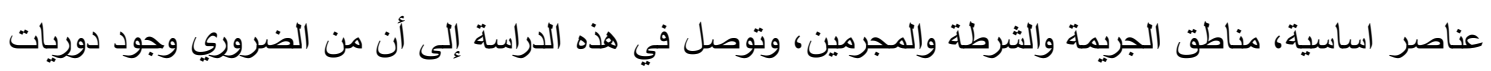

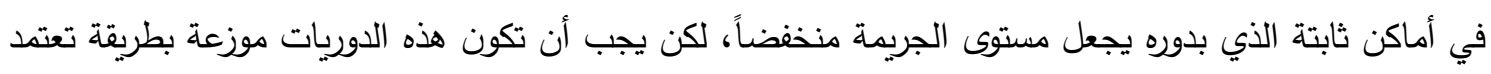

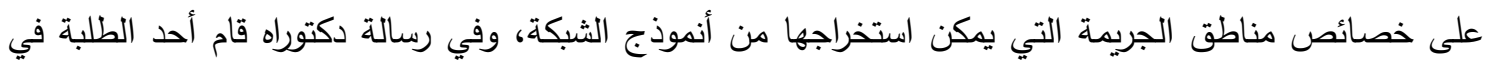

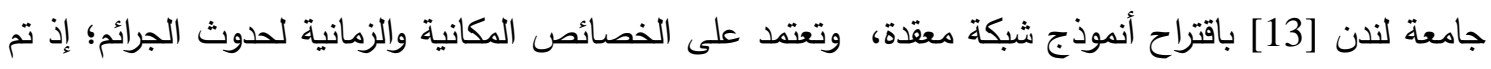



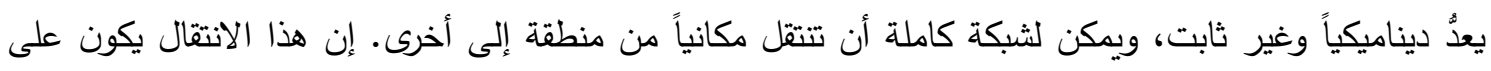

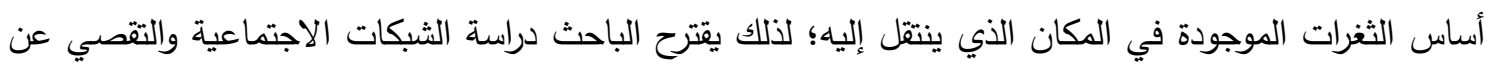
وجود هكذا حالات، وفي دراسة عن الثبكات الإجرامية للمهاجرين في الدول المتقدمة، قام الباحث نوزينا [14]

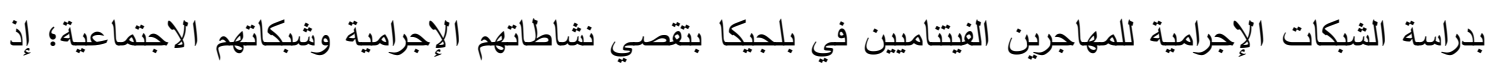



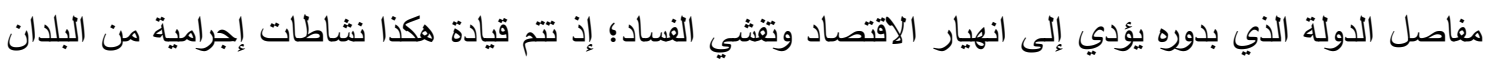
الأم للمهاجرين، مثال آخر على نشاطات المهاجرين هي حالة المهاجرين النيجيرين في الولايات المتحدة الامريكية؛ إذ قام الباحث جونز [15] بتحليل النشاطات التي يقوم بها المهاجرون النيجيريون، وقد وجد أن أغلب الجرائم التي

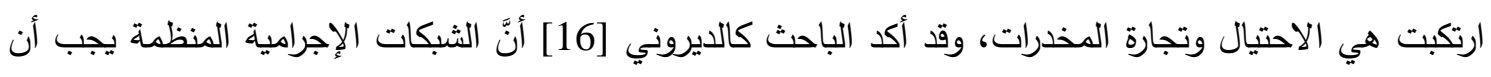
تدرس بطريقتين الأولى: دراسة هيكلية الثبكة، والثانية: هي دراسة العلاقات والخواص الاجتماعية لهذه الثبكات.

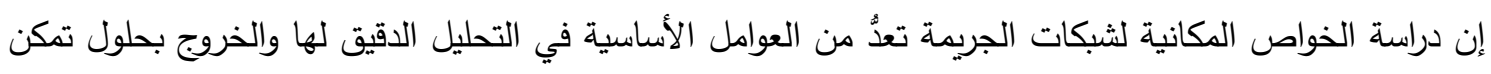

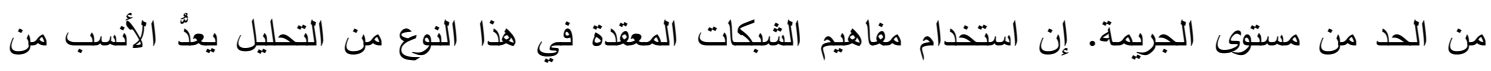

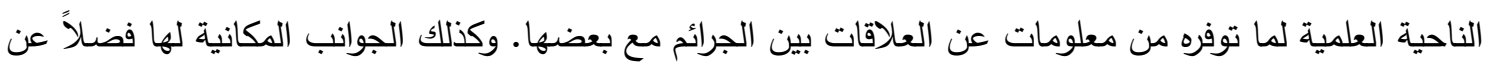


الجوانب الزمانية ومعايير قياس الثبكة كما أثبت في [17] و[18] و[19] مما يجعل استخدام هذه الطرائق من التوجهات الحديثة في مجال علم تحليل الجريمة. 3. طريقة البحث 1.3

إن عملية جمع البيانات تعدُّ من أهم المراحل في هذا البحث؛ إذ إنها تعدّ المادة التي سوف نقوم بدراستها وتحليلها، ولم تكن عملية جمع البيانات في الحقيقة بالمهمة السهلة؛ والسبب أن هكذا بيانات تحتاج إلى مخاطبات وموافقات رسمية للحساسية التي تتمتع بها البيانات، بعد استحصال جميع الموافقات الرسمية ومفاتحة عمادة كلية علوم الحاسوب والرياضيات ورئاسة جامعة الموصل للجهات المسؤولة في وزارة الداخلية تم الحصول على البيانات الخام للجرائم المرتكبة في محافظة نينوى، وقد تمت مفاتحة كل من رئاسة استئناف محكمة نينوى الاتحادية، ومديرية شرطة محافظة نينوى والمنشآت/ قسم الإحصاء، ومديرية شرطة محافظة نينوى المنشات/ مديرية الجنائية

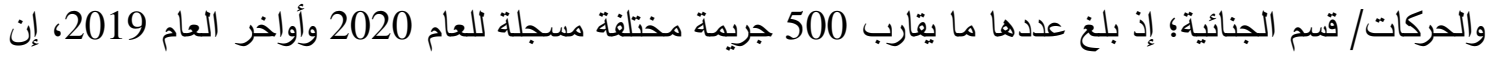
هذه الكمية من البيانات تعد كافية جداً للقيام بإجراء الدراسة والبحث والتحليل، ويجدر بالذكر أن منتسبي قسم الجنائية قدموا جميع التسهيلات اللازمة طوال أيام جمع البيانات، إن المشكلة الأخرى التي واجهتا أثناء عملية

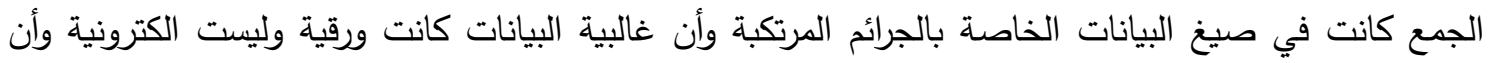
بعضها تطلب صياغة معينة لكي تمثل في الثبكة المعقدة، كذلك كان هنالك بيانات منقوصة الصيغة التي احتاجت الكثير من الجهد اليدوي والوقت لإكمالها. بعد إجراء كل ما سبق قمنا بصياغة البيانات لكي تكون ملائمة لغرض التمثيل وتكوين الثبكة كما في لهي الجدول رقم 1، إن الصيغة التي خُولت البيانات إليها هي خاصة بالثبكات المعقدة التي تبرمج على مرحلتين، الأولى خاصة بالعقد، والثانية خاصة بالحواف التي تربط العقد، حقيقة هناك عدة صيغ بتمثيل الثبكات المعقدة لكن الصيغة التي اعتمدنا عليها هي صيغة ملفات التهد، GEXF [32] التي تستخدم في برنامج Gephi [33]، إن أبرز حقول البيانات التي جمعت تتضمن: 1- نوع الجريمة المرتكبة (سرقة، احتيال، قتل، الخ) 2- تاريخ ارتكاب الجريمة (يوم وشهر وسنة). 3- محل سكن الجاني أو مرتكب الجريمة، هنا يجب ملاحظة أن البيانات التي جمعت لا تلتضمن معلومات عن

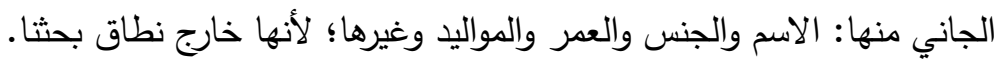
4- موقع منطقة حدوث الجريمة إذا كان في مدينة الموصل (الأيمن أو الأيسر) 5- اسم القضاء أو الناحية إذا كانت الجريمة مرتكبة في أحد أقضية أو نواحي الدحافظة الدئة الدينة

الجدول (1): صيغة البيانات

\begin{tabular}{|c|c|c|c|c|c|c|c|}
\hline جميع المناطق & خدارج الجرائم المرتكبة & خارج/داخل الجاني من & داخل المنطقة & جانب المدينة & مدينة الموصل/خارج & المنطقة & الجريمة \\
\hline
\end{tabular}


لقد اعتمد على البيانات التي جمعت في تكوين الثبكة، إن الثبكة الإجرامية التي كونت تضم عقداً وحوافٍ تربطها. كما أسلفنا سابقاً، قمنا بتكوين شبكتين في هذا البحث وعلى النحو الآتي:

شبكة الجرائم:

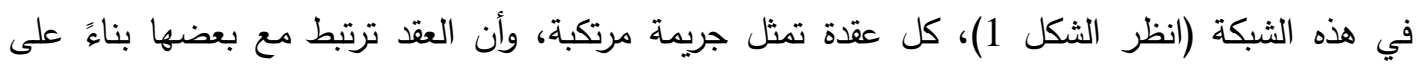


أنواع الحواف فيها من نوع غير الموجه، إن هذه الطريقة في ربط عقد الجرائم قد اتبعت في العديد من الدراسات

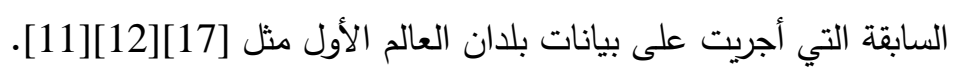

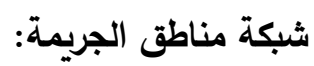

هذه الشبكة (انظر الشكل 2) تختلف في هيكليتها عن الشبكة السابقة؛ إذ إن كل عقدة فيها تمثل منطقة معينة من المناطق التي ارتكبت فيها الجرائم، وأن كل منطقتين ترتبطان حسب استراتيجية معينة وهي على النحو الآتي: ترتبط عقدتان إذا حصلت فيهما نوع الجريمة نفسها.

• يحدد اتجاه الحافة إذا كان مرتكب الجريمة من منطقة (عقدة) وقام بجريمة في منطقة أخرى (عقدة أخرى).

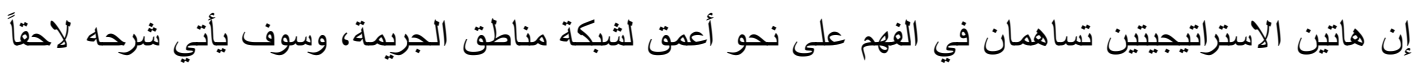



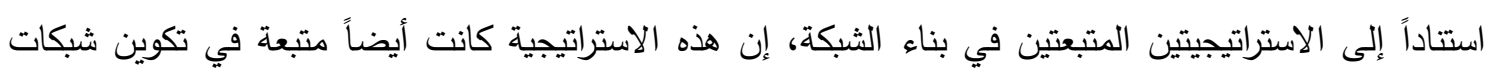
الجريمة في الدراسات السابقة مثل [18][13][13]. إن عملية التحليل واستخراج النتائج سوف تكون لكل شبكة على حدة، كذلك سوف نخرج بالعديد من الاستتناجات بناءً على الشبكتين المكونتين.



الثكل 1: شبكة الجرائم المرتكبة (كل لون يمثل جريمة معينة). 


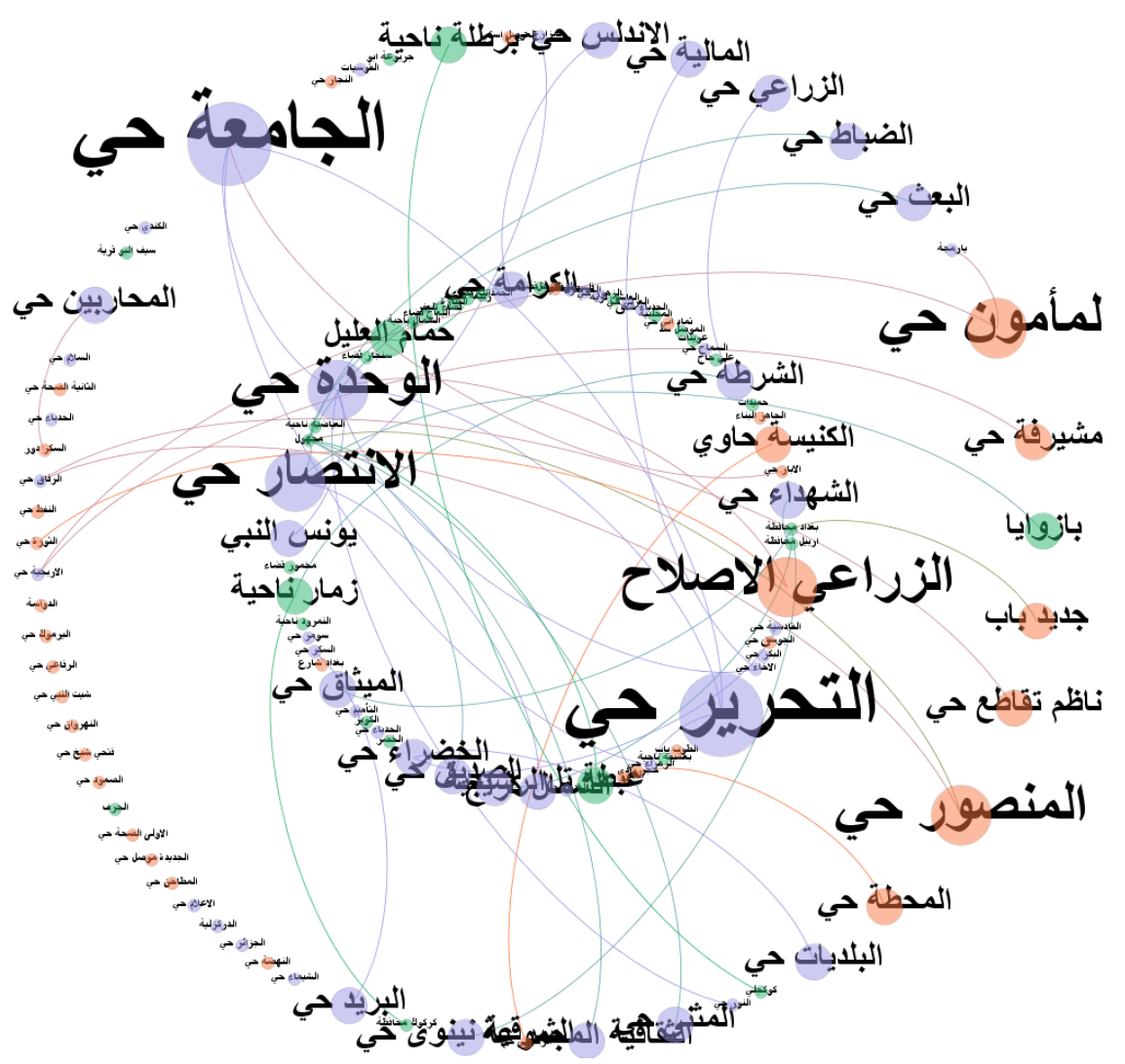

الثكل 2: شبكة مناطق الجريمة (اللون الأزرق يمثل مناطق الجانب الأيسر لددينة الموصل واللون البرتقالي يمثل الجانب الأيمن واللون الأخضر يمثل أقضية محافظة نينوى ونواحيها).

4. النتائج والمناقشة 1.4

بعد تكوين شبكة الجرائم قمنا بتحليلها على أساس معايير الثبكة المركزية، وفي البدء نستعرض في الثكل (3) النسب المؤية لأنواع الجرائم في محافظة نينوى؛ إذ نشاهد أن أعلى نسبة هي جرائم الانتحار ، ولم نتوقع في



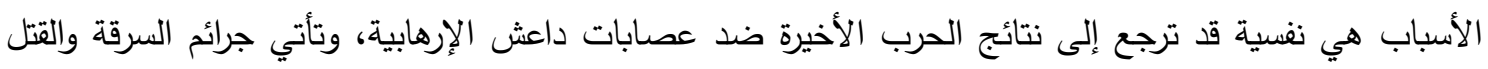
العمد في مقدمة الجرائم بعد الانتحار، ومن المفاجئ ظهور بعض الجرائم التخريبية المرتكبة مثل جرائم حرق الإهياته

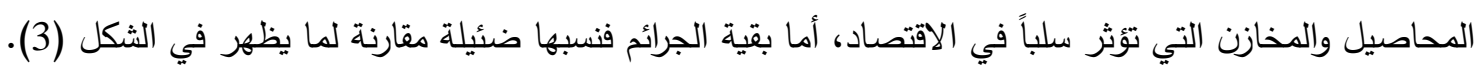




\section{النسبة المؤية}

\section{انتحار}



\section{خدام: \\ الثكل (3): النسب المؤية لأنواع الجرائم في محافظة نينوى}

إن أول خطوة في تحليل شبكة الجرائم هي استخراج خصائص الثبكة العامة والجدول (2) يظهر هذه

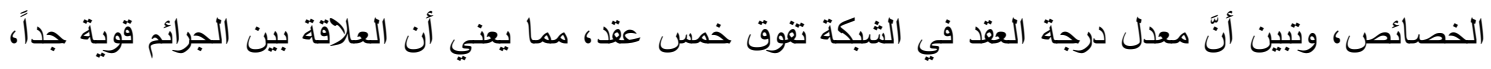

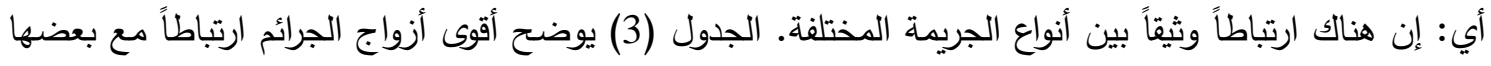



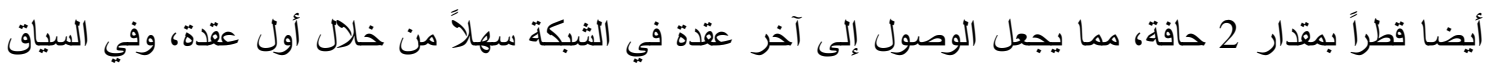

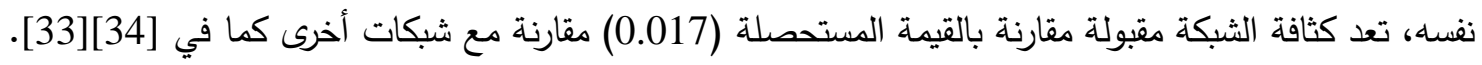
نلاحظ أيضاً أن معامل الارتباط أو العنقدة للشبكة عالٍ جداً والذي بدوره يعكس معدل درجة العقدة للشبكة وكثافتها،

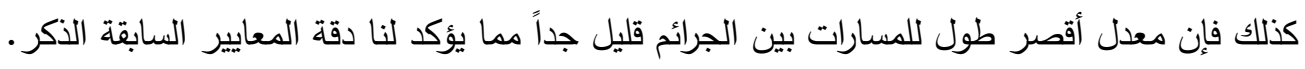


الجدول (2): خصائص شبكة الجرائم

معدل درجة العقد المركزية قطر الثبكة كثافة الثبكة معدل معامل العنقدة معدل اقصر طول للمسارات

الجدول (3): ازواج الجرائم الأكثر ارتباطاً في شبكة الجريمة

\begin{tabular}{|c|c|c|}
\hline قيمة الارتباط & الزوج & التسلسل \\
\hline 0.763 & سرقة - قتل عمد & 1 \\
\hline 0.701 & انتحار - اعتداء & 2 \\
\hline 0.7 & مشاجرة - شروع بالقتل & 3 \\
\hline 0.684 & سرقة - حيازة غير قانونية & 4 \\
\hline 0.495 & احتيال - تزوير & 5 \\
\hline 0.412 & اعتداء - حرق & 6 \\
\hline 0.388 & خطف - انتحار & 7 \\
\hline
\end{tabular}

وفضلاً عمَّا ذكر قمنا باستخدام معايير الثبكة المركزية في تحليل شبكة الجرائم؛ إذ رتبت أسبقيات الجرائم حسب موقعها في هيكل الثبكة عن طريق معيار البينية المركزية، ويقوم هذا المعيار ببيان جريمة معينة من الجرائم في الشبكة وتأثيرها في بقية الجرائم، أي يظهر مدى مركزية جريمة معينة بين الجرائم. الجدول (4) يظهر أعلى ترتيب للجرائم من ناحية التمركز في الثبكة، ويحتوي الجدول أيضاً على معيار القرب المركزي الذي يعكس مدى هـى قرب جريمة معينة من بقية الجرائم، إنَّ الجدول (4) يبين أربعة أنواع من الجرائم التي تعدُّ مركزاً لجميع الجرائم في لهي

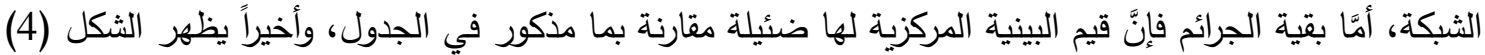
ارتباط جميع الجرائم مع بعضها في الثبكة. الجدول (4): الجرائم ذات الأعلى بينية مركزية وقرب مركزي

\begin{tabular}{|c|c|c|}
\hline معيار القرب المركزيي & معيار البينية المركزية & نوع الجريمة \\
\hline 1.00 & 0.00065 & خطف \\
\hline 1.00 & 0.00063 & حرق محاصيل/مخازن/متاجر \\
\hline 0.75 & 0.00048 & 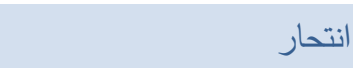 \\
\hline 0.75 & 0.00036 & 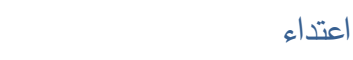 \\
\hline
\end{tabular}






الثكل (4): ارتباط الجرائم مع بعضها البعض في شبكة الجريمة، كل لون يمثل نوعاً من الجريمة وأن الجرائم من النوع نفسه مبينة على شكل مجموعة أو عنقود يرتبط مع بقية المجاميع في الثبكة.

2.4 نتائج شبكة مناطق الجريمة

قمنا بعد تكوين شبكة مناطق الجريمة باستخراج خصائصها وحسب معايير الثبكة المستخدمة في شبكة

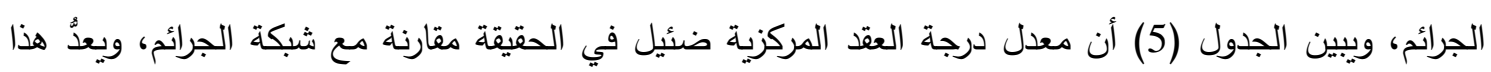

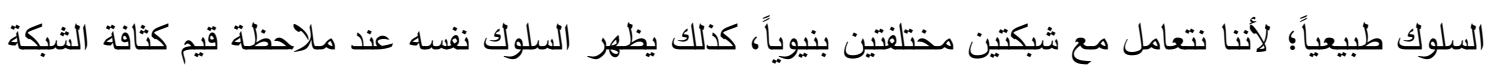

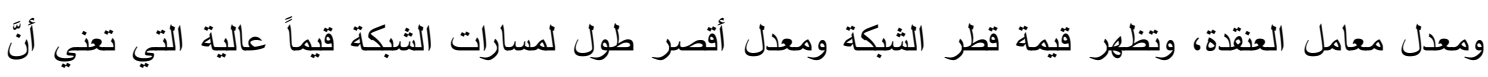
العلاقات بين المناطق تظهر سلوكاً يدل على قصر المسار من منطقة إلى أخرى عن طريق جريمة معينةً. الجدول (5): خصائص شبكة مناطق الجريمة

\begin{tabular}{|c|c|c|c|c|}
\hline معدل طول المسارات & معدل معامل العنقدة & كثافة الثبكة & قطر الشبكة & معدل درجة العقد المركزية \\
\hline 1.297 & 0.009 & 0.003 & 3 & 0.383 \\
\hline
\end{tabular}

قمنا أيضاً بالتقصي عن المناطق الأكثر تعرضاً للجريمة من خلال درجات العقد المركزية في الثبكة، الثكل

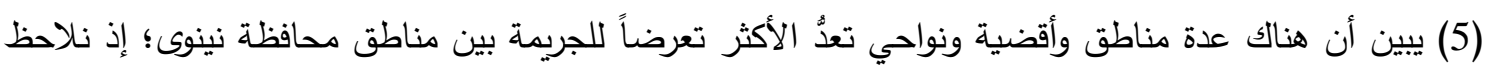



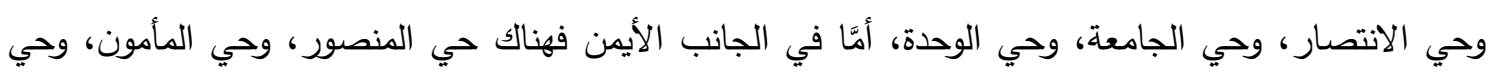


الإصلاح الزراعي، وهي المناطق الأبرز في التعرض للجريمة، أمَّا الأقضية والنواحي التابعة لمحافظة نينوى فإن ناحية زمار ، وناحية برطلة، وتل عبطة، وناحية حمام العليل، وبازوايا هي المناطق الأبرز في تلقي الجريمة خارج

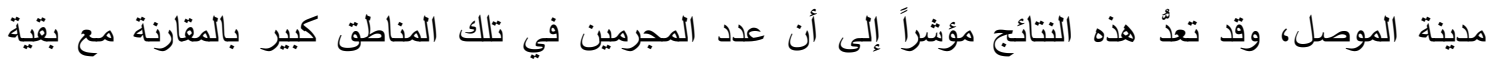
المناطق؛ لهذا السبب قمنا بالتقصي عن المناطق التي تحتوي على أكبر عدد من الجناة، أي التقصي عن مرتكبي الجرائم في المناطق. ويظهر الثكل (6) المناطق الأكثر احتواءً للجناة؛ إذ يتبين أن هناك مناطق تعدُ مصدراً

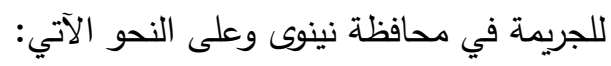
• الجانب الأيسر يضم الأحياء الآتية: حي الوحدة، وحي الانتصار، وحي الجامعة، وحي التحرير، وحي الكرامة،

$$
\text { وحي الإخاء، والأربجية، وحي الزهراء. }
$$

• الجانب الأيمن يضم الأحياء الآتية: حي المنصور، واهي، وحي المأمون، وحي الإصلاح الزراعي، وحي الزنجيلي،

$$
\text { وموصل الجديدة، وحاوي الكنيسة، وحي الثورة. }
$$

• الأقضية والنواحي تضم المناطق الآتية: ناحية زمار، وناحية ربيعة، وناحية برطلة، وكوكجلي، وبازوايا، وتل

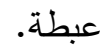
• المحافظات الأخرى: بغداد، أربيل، بابل، كركوك.

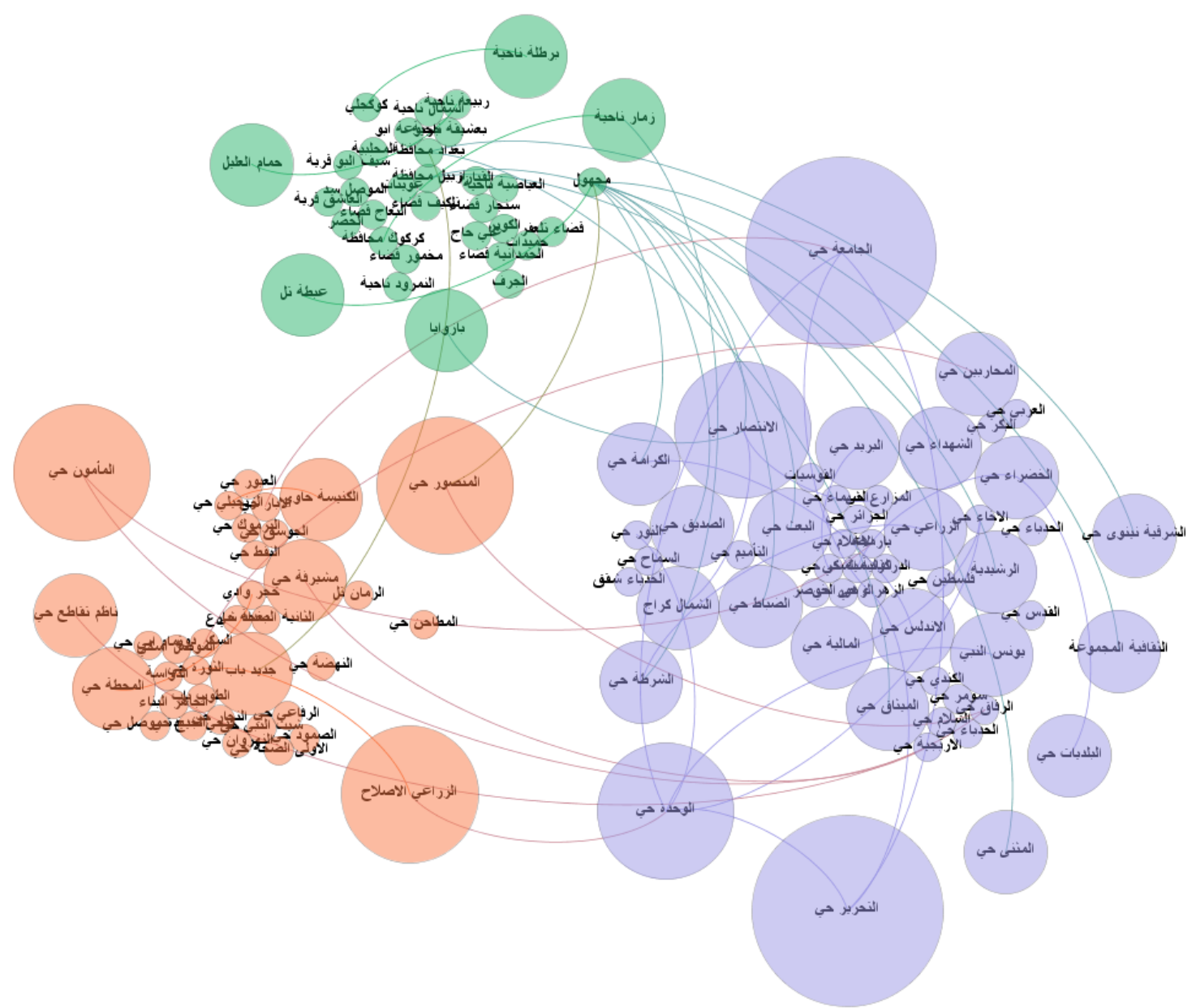

الثكل (5): شبكة مناطق الجريمة التي تبين المناطق الأكثر تعرضاً للجريمة في محافظة نينوى، اللون الأزرق للعقد إلى يمين

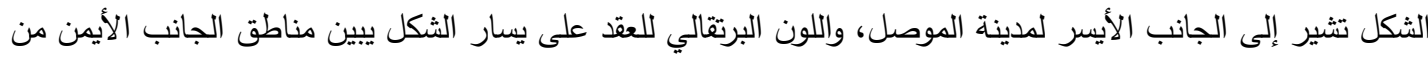






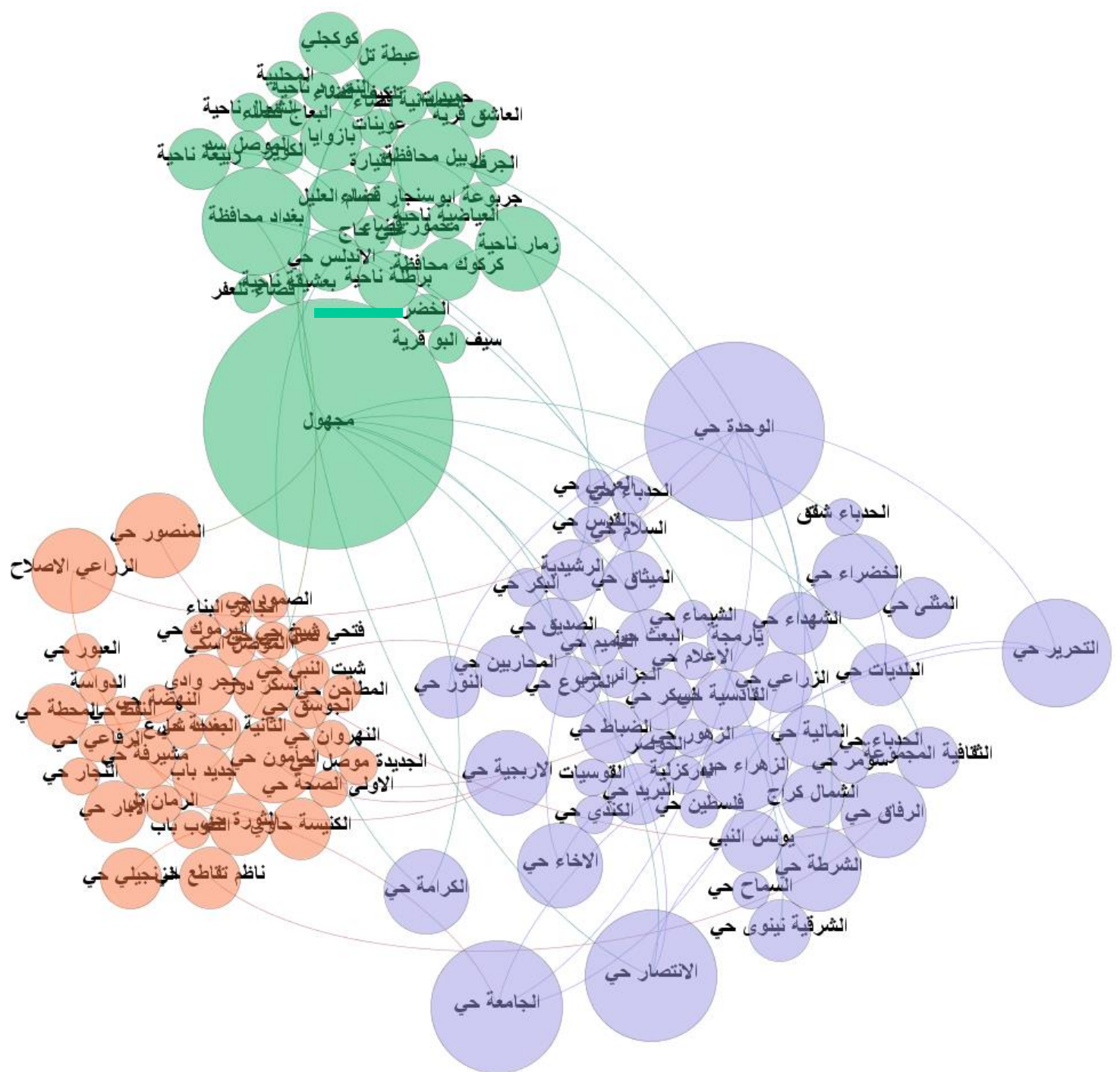

الثكل (6): شبكة مناطق الجريمة التي تبين المناطق التي تحتوي على أكبر عدد من الجناة في محافظة نينوى، واللون

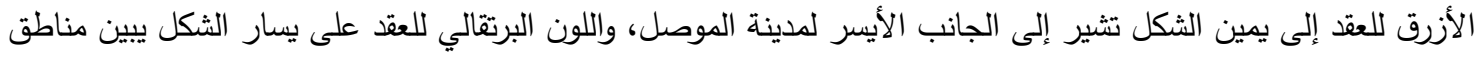

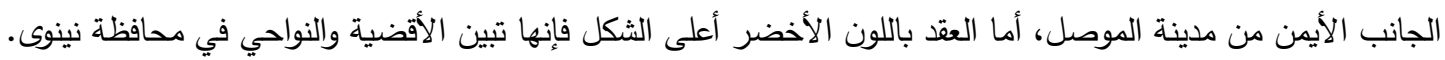

لقد تم التقصي ايضاً عن عدد الجرائم في أحياء مدينة الموصل، وقد أظهرت النتائج الإحصائية أن أكثر المناطق احتواءً للجريمة من ناحية عدد الجرائم وأن مرتكبيها من المنطقة نفسها هي: حي الكرامة، وحي الوحدة،

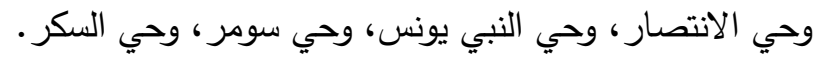

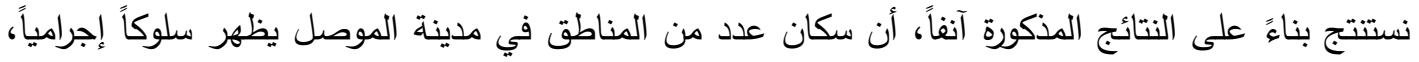
وعليه قمنا بالتقصي عن ميول تلك المناطق في نقل سلوكها إلى المناطق الأخرى؛ إذ يمكن قياس هذه الميول

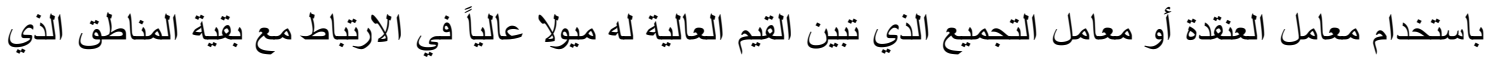
يؤدي بدوره إلى نسخ السلوك، الجدول (6) يبين هذه القيم والمناطق المحتملة أن تتبع هكذا سلوك. 
الجدول (6): المناطق الأعلى معامل عنقدة (لايها ميل لنسخ سلوكها إلى باقي المناطق)

معامل العنقدة

من خلال الجدول أعلاه يتبين أن هناك أجزاء من مدينة الموصل تعد الأخطر على المدينة من ناحية سلوك

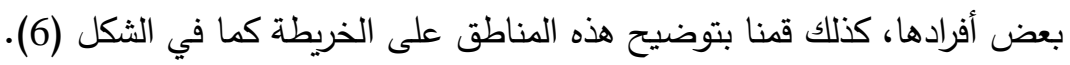

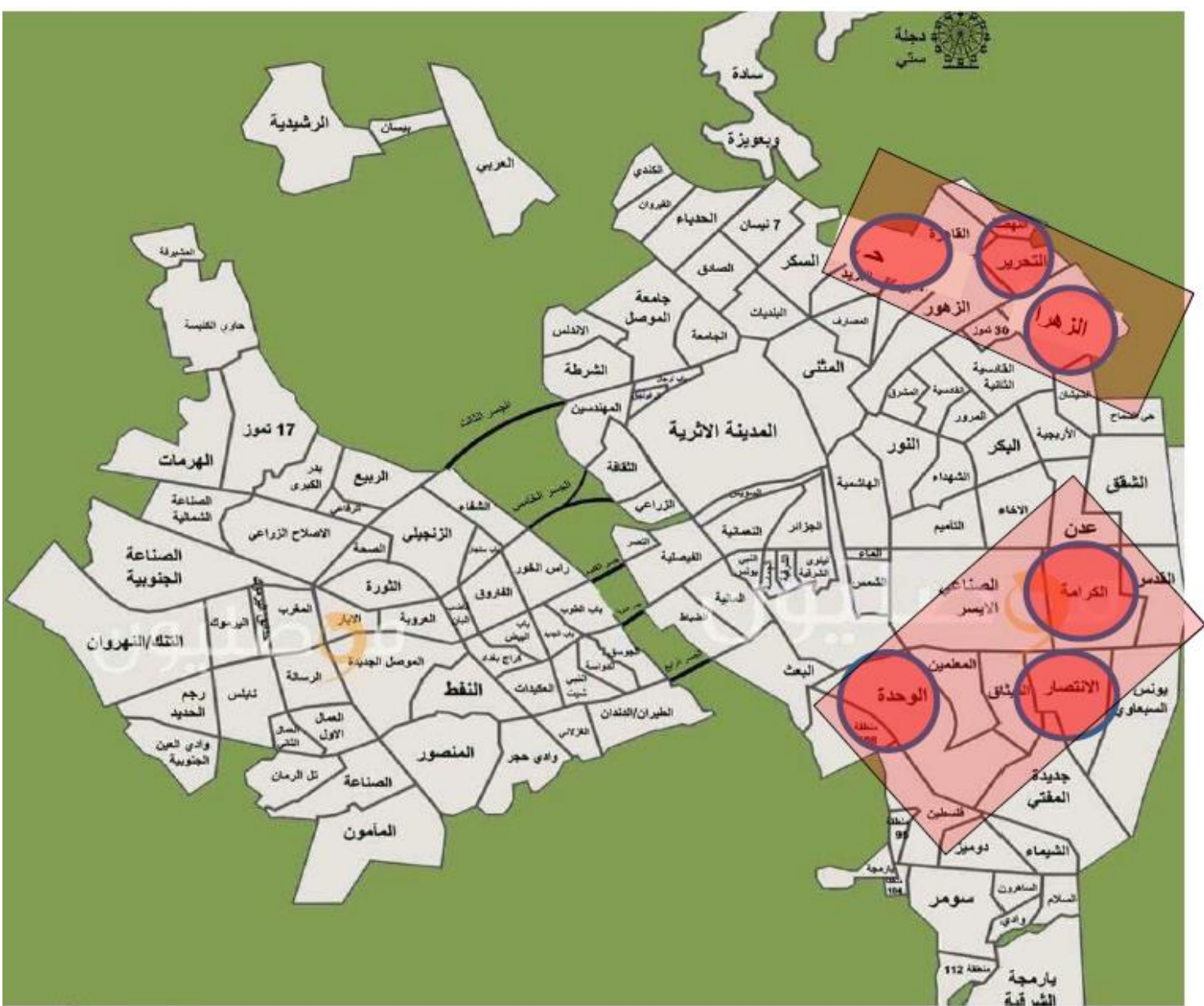

الثكل (6): خريطة مدينة الموصل توضح المناطق التي تعد الأكثر خطراً من ناحية بعض ساكنيها من المجرمين.

يتبين من الثكل أعلاه أن هناك منطقتين رئيستين في الجانب الشرقي لمدينة الموصل كل منها تضم ثلاثة أحياء التي يجب أخذها بنظر الاعتبار عند التطرق لموضوع الجريمة. علماً أن ميول هذه المناطق لتكوين

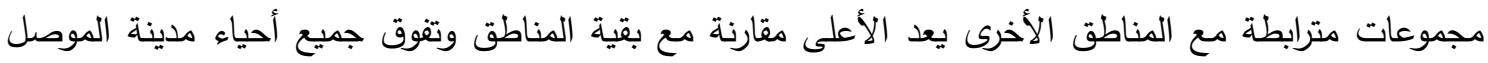
بجانبيها الأيسر والأيمن في هذا الخصوصن

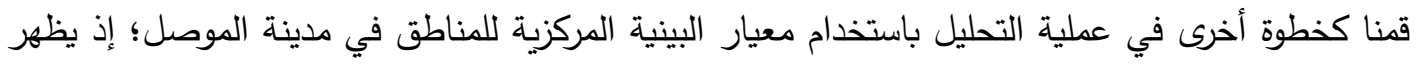
هذا المعيار مدى تمركز منطقة معينة في شبكة الجريمة في مدينة الموصل. الجدول (7) يظهر المناطق الأكثر

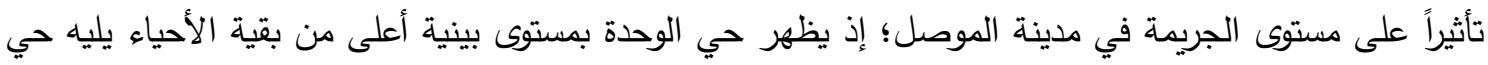




مناطق موجودة في الخارطة أعلاه، وتظهر معها كذلك المناطق المحاذية لها، تؤكد هذه النتيجة على ما تم ذكر في الفقرات السابقة فيما يخص نسخ السلوك؛ إذ تظهر أحياء الميثاق والخضراء وهي محاذية للمناطق ذات المعات معامل


المحافظات الأخرى والأقضية والنواحي، وهذا ما يجعل منها منطقة خطرة لما تحويه من كثافة سكانية تمتزج فيها

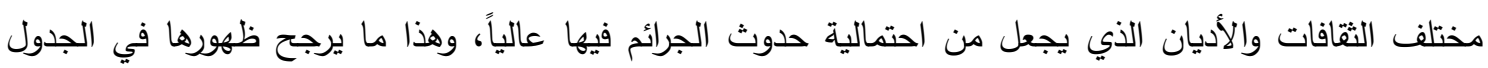

\begin{tabular}{|c|c|c|c|c|c|c|c|}
\hline الميثُاق & الخضراء & الجامعة & الكرامة & الثرطة & الانتصار & الوحدة & المنطقة \\
\hline 0.000071 & 0.000076 & 0.000075 & 0.000078 & 0.000141 & 0.00028 & 0.00057 & المركزية \\
\hline
\end{tabular}

يتبين بناء على ما ورد من نتائج أن الأجزاء الثرقية لجانبي مدينة الموصل الأيمن والأيسر هي الأكثر خطورة على المدينة. الثكل (7) يوضح خارطة مدينة الموصل بجانبيها مع مستوى الخطورة لكل جانب (مؤشرة بالتدرج الأحمر).

من الجدير بالذكر أن هناك ارتباطاً ما بين جانبي المدينة الأيسر والأيمن من ناحية الجريمة، وكذلك مع الألك الأقضية والنواحي، مثال على ذلك هناك جناة من منطقة حي الوحدة قاموا بارتكاب جرائم في جانب المدينة الأيمن،




بعد إظهار النتائج هناك مجموعة من التوصيات نود طرحها وهي على النحو الآتي:

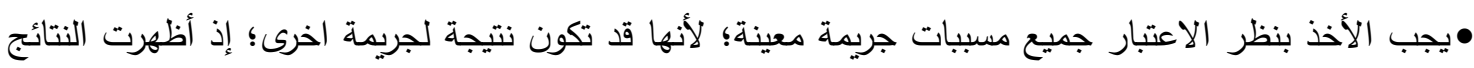
ارتباطاً قوياً بين الجرائم.

• يجب اتباع استراتيجيات خاصة مع مناطق معينة للحد من نسبة الجريمة فيها، وكذلك إيقاف تصديرها للجريمة. • هناك مناطق في مدينة الموصل تعد الأكثر خطراً على مستوى الجريمة في المحافظة، لذلك من الضروري

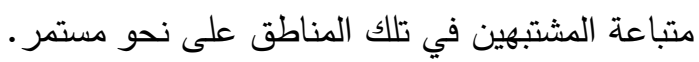
•يجب التركيز أيضاً على المناطق المحاذية للمناطق الخطرة والحد من انتقال الجريمة إليها.

\section{5. الاستنتاجات والأعمال المستقبلية}

تم دراسة الثبكات الإجرامية وحللت على وفق مفاهيم الثبكات المعقدة؛ إذ كونت شبكتان، الأولى تمثل أنواع الجريمة، والثانية تمثل مناطق الجريمة؛ استتاداً إلى النتائج التي تم الحصول علئي عليها، نستتتج أن مفاهيم الثبكات المعقدة تعد أداة فعالة في تحليل الثبكات الإجرامية؛ لأنها تتقصى العلاقات بين أجزاء بيانات الجريمة، كذلك تعطي استخدام معايير قياس الثبكة مؤشرات مهمة على طبيعة الشبكة من الناحية الهيكلية مما يسهل عملية التحليل

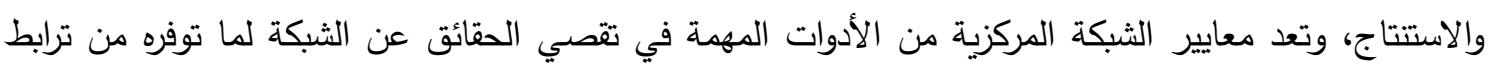

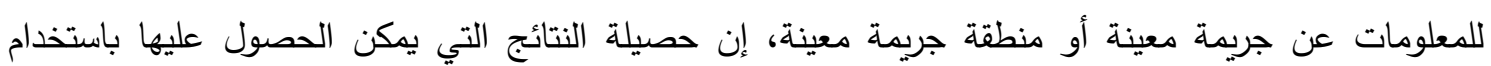
مفاهيم الثبكات المعقدة يمكن أن تستخدم في الخروج بتوصيات عن الطرائق والاسترايجيات التي يمكن اتباعها للحد 
إن الأعمال المستقبلية لهذا البحث يمكن أن يوظف وتمتد فعالياته ليطبق في جميع أنحاء العراق لتشمل شبكة الجريمة بأنواعها، وهذه العملية تحتاج إلى قاعدة بيانات يمكن أن توفرها وزارة الداخلية، وأن الثبكة التي أنشئت والتي تمثل شبكة الجريمة في العراق قد تعطي مؤشرات معتمدة للحد من مستوى الجريمة فضلاً عن تحديد مواطن الخلل في الجوانب الأمنية المتعلقة بالجريمة، ونروم أيضاً في أعمالنا المستقبلية التركيز على الجرائم الالكترونية التي تحدث على نحو كبير في شبكات التواصل الاجتماعي التي تثتمل على العديد من الجرائم كالابتزاز والتهديد والتشهير






$$
\begin{aligned}
& \text { المصادر } \\
& \text { ياسر عامر عمران المختار • (2020). جدلية تعريف جريمة العدوان في القانون الدولي العام. مجلة رسالة } \\
& \text { الحقوق, 12 1(1), 316-316. } \\
& \text { هاجر زهران. (2013). تعريف الجريمة، وبيان أصلها، وأنواعها، وطرق إثباتها. Al-Madinah } \\
& \text { /Quranic Studies Studies } \\
& \text { العنزي, عامر بن قيران مسدح, عبد المحمود, \& عباس أبو شامة. مشرف. (2020). آليات التعاون في } \\
& \text { مكافحة الجريمة المنظمة: دراسة على ضوء إستراتيجية مجلس الوزراء الداخلية العرب: دراسة تحليل } \\
& \text { مضدون Doctoral dissertation, جامعة نايف العربية للعلوم الأمنية). } \\
& \text { عادل يوسف عبد النبي الثكري. (2019). الشرط المفترض وموضعه في الانموذج القانوني للجريمة } \\
& \text { (دراسة تحليلية في البنية القانونية للجريمة). مجلة الكوفة للعلوم القانونية والسياسية , 1(39), 27-46. }
\end{aligned}
$$

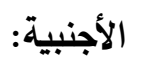

[5] Furtado, V., Melo, A., Coelho, A. L., Menezes, R., \& Perrone, R. (2009). A bioinspired crime simulation model. Decision Support Systems, 48(1), 282-292.

[6] Oliveira, M., Barbosa-Filho, H., Yehle, T., White, S., \& Menezes, R. (2015). From criminal spheres of familiarity to crime networks. In Complex Networks VI (pp. 219-230). Springer, Cham.

[7] White, S., Yehle, T., Serrano, H., Oliveira, M., \& Menezes, R. (2014, November). The spatial structure of crime in urban environments. In International Conference on Social Informatics (pp. 102-111). Springer, Cham.

[8] Wang, P., Yang, Z., Niu, S., Zhang, Y., Zhang, L., \& Niu, S. (2018, June). Modeling dynamic pairwise attention for crime classification over legal articles. In The 4lst International ACM SIGIR Conference on Research \& Development in Information Retrieval (pp. 485-494).

[9] Toledo, A. S. O., Carpi, L. C., \& Atman, A. P. F. (2020). Diversity Analysis Exposes Unexpected Key Roles in Multiplex Crime Networks. In Complex Networks XI (pp. 371-382). Springer, Cham.

[10] Rossy, Q., \& Morselli, C. (2018). The contribution of forensic science to the analysis of crime networks. The Routledge International Handbook of Forensic Intelligence and Criminology, 191-204.

[11] Oliveira, M., \& Menezes, R. (2019). Spatial concentration and temporal regularities in crime. arXiv preprint arXiv:1901.03589.

[12] Melo, A., Menezes, R., Furtado, V., \& Coelho, A. L. (2006, September). Selforganized and social models of criminal activity in urban environments. In International Workshop on Ant Colony Optimization and Swarm Intelligence (pp. 518-519). Springer, Berlin, Heidelberg.

[13] Zolghadriha, S. (2018). The Investigation of Organised Crime Networks (Doctoral dissertation, UCL (University College London)). 
[14] Nožina, M. (2010). Crime networks in Vietnamese diasporas. The Czech Republic case. Crime, law and social change, 53(3), 229-258.

[15] Jones, M. (1993). Nigerian crime networks in the United States. International journal of offender therapy and comparative criminology, 37(1), 59-73.

[16] Calderoni, F. (2019). 29 Transnational Organized Crime Networks. International and Transnational Crime and Justice, 175.

[17] Oliveira, M., Bastos-Filho, C., \& Menezes, R. (2017). The scaling of crime concentration in cities. PloS one, 12(8), e0183110.

[18] Oliveira, M., Ribeiro, E., Bastos-Filho, C., \& Menezes, R. (2018). Spatiotemporal variations in the urban rhythm: the travelling waves of crime. EPJ Data Science, 7(1), 29.

[19] Campana, P., \& Varese, F. (2020). Studying organized crime networks: Data sources, boundaries and the limits of structural measures. Social Networks.

[20] Bollobás, B. (2013). Modern graph theory (Vol. 184). Springer Science \& Business Media.

[21] West, D. B. (1996). Introduction to graph theory (Vol. 2). Upper Saddle River, NJ: Prentice hall.

[22] Deo, N. (2017). Graph theory with applications to engineering and computer science. Courier Dover Publications.

[23] Foulds, L. R. (2012). Graph theory applications. Springer Science \& Business Media.

[24] Evsukoff, A. (2013). Complex Networks. R. Menezes, \& M. C. González (Eds.). Springer.

[25] Ghoshal, G., Mangioni, G., Menezes, R., \& Poncela-Casanovas, J. (2014). Social system as complex networks.

[26] Barabási, A. L. (2013). Network science. Philosophical Transactions of the Royal Society A: Mathematical, Physical and Engineering Sciences, 371(1987), 20120375.

[27] Albert, R., \& Barabási, A. L. (2002). Statistical mechanics of complex networks. Reviews of modern physics, 74(1), 47.

[28] Barabási, A. L. (2009). Scale-free networks: a decade and beyond. science, 325(5939), 412-413.

[29] Strogatz, S. H. (2001). Exploring complex networks. nature, 410(6825), 268276.

[30] Boccaletti, S., Latora, V., Moreno, Y., Chavez, M., \& Hwang, D. U. (2006). Complex networks: Structure and dynamics. Physics reports, 424(4-5), 175-308.

[31] Ravasz, E., \& Barabási, A. L. (2003). Hierarchical organization in complex networks. Physical review E, 67(2), 026112.

[31] Heidler, R., Gamper, M., Herz, A., \& Eßer, F. (2014). Relationship patterns in the 19th century: The friendship network in a German boys' school class from 1880 to 1881 revisited. Social Networks, 37, 1-13. 
[32] Bastian, M., Heymann, S., \& Jacomy, M. (2009). Gephi: an open source software for exploring and manipulating networks. Icwsm, 8(2009), 361-362.

[33] Dong, K., Benson, A. R., \& Bindel, D. (2019, July). Network density of states. In Proceedings of the 25th ACM SIGKDD International Conference on Knowledge Discovery \& Data Mining (pp. 1152-1161).

[34] Krumov, T. (2019). Determination of the optimal density of the forest road network. Journal of Forest Science, 65(11), 438-444. 